\title{
Mediastinal teratoma presenting as apparent fresh stillbirth
}

Neonatal teratomas, the most common perinatal neoplasm, are usually benign with the sacrococcygeal region being the commonest site. ${ }^{1}$ We report a case of a mediastinal teratoma presenting as an apparent fresh stillbirth.

A female infant, birth weight $3.23 \mathrm{~kg}$, was born by normal delivery to a 36 year old primigravida at 36 weeks gestation. The pregnancy had been uneventful, and a 19 week fetal anomaly scan was normal. A fetal heart was detected by cardiotocograph just before delivery; however, at birth there were no signs of life and the baby had evidence of mild hydrops. The infant was intubated immediately and received full resuscitation. Adrenaline (epinephrine) and colloid were administered in the course of the resuscitation, and a heart beat was first detected by 15 minutes of age. The heart rate exceeded 100 beats per minute by 18 minutes of age. An initial arterial blood gas assessment showed a $\mathrm{pH}$ of $6.43, \mathrm{PCO}_{2}$ of 200.3 $\mathrm{mm} \mathrm{Hg}, \mathrm{PO}_{2}$ of $25.2 \mathrm{~mm} \mathrm{Hg}$, bicarbonate of $12.5 \mathrm{mmol} / 1$, and base deficit of $25.1 \mathrm{mmol} / 1$. A chest radiograph disclosed a large anterior mediastinal mass (fig 1). A spiral computed tomography scan of the chest performed at 6

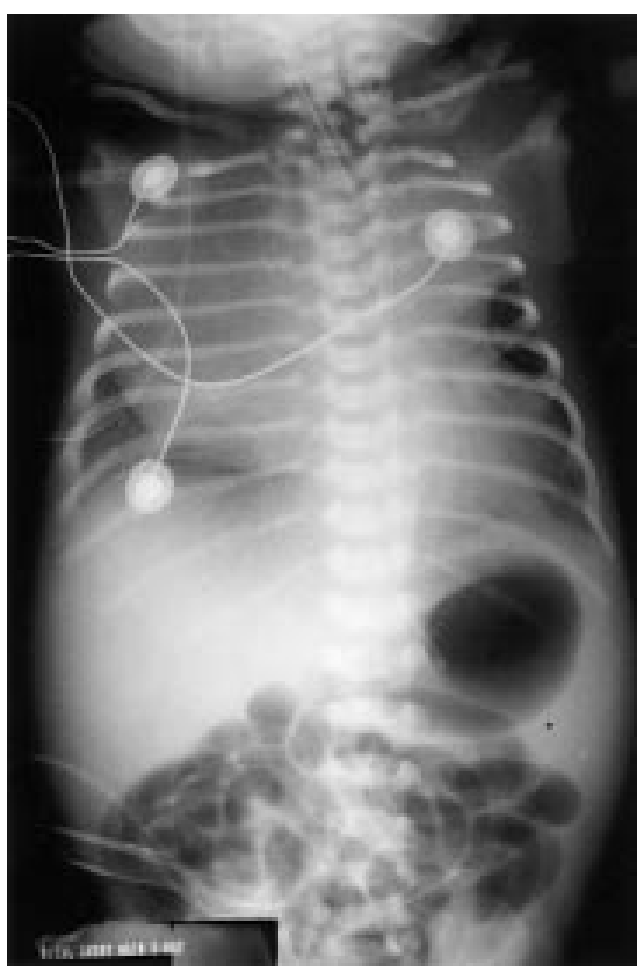

Figure 1 Radiograph showing mediastinal mass.

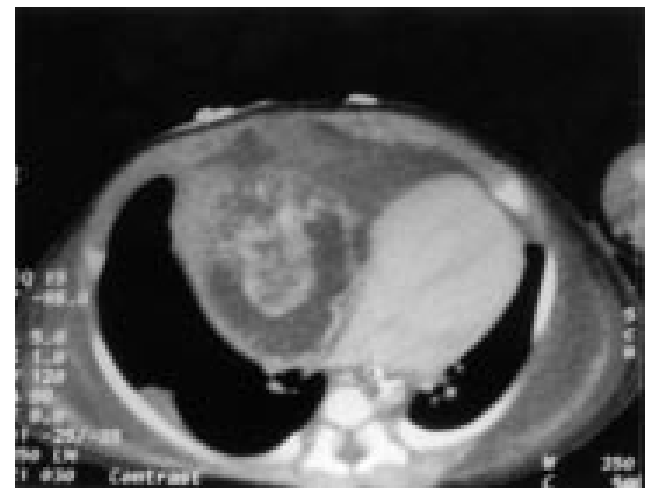

Figure 2 Computed tomography scan of mass showing areas of altered attenuation within the mass and massive displacement of mediastinal structures.

hours of age confirmed a large, oval, well defined mass extending downwards from the thoracic inlet anteriorly to the base of the chest. The mass contained cystic and solid elements, and the appearances were consistent with a teratoma (fig 2).

Surgery was performed on day 3 of life. A well encapsulated tumour lying behind the sternum was excised, complete and intact. Histological examination showed a mass weighing $104 \mathrm{~g}$, and the appearances were those of an immature mediastinal teratoma (fig 3). The infant was discharged home, well, on day 16 of life. Subsequent follow up has shown normal developmental progress at 3 years of age.

Teratomas can present in a wide variety of locations with diverse clinical behaviour. Mediastinal teratomas account for $4-11 \%$ of childhood teratomas. ${ }^{2}$

Mediastinal teratomas in infancy usually cause compression of vital structures. Our case illustrates an extreme example of this and shows that normal survival can be achieved even after aggressive respiratory and cardiovas-

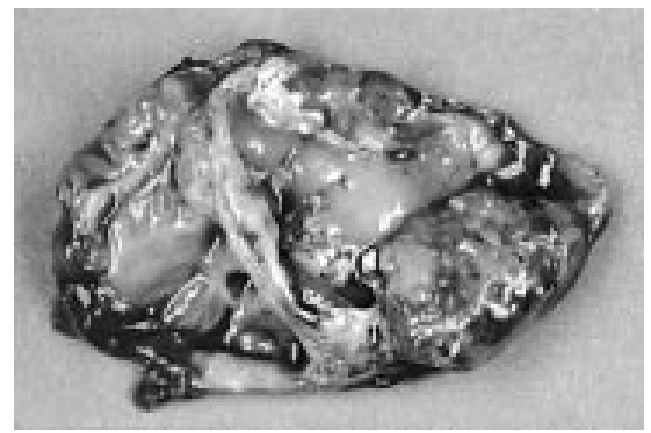

Figure 3 Macroscopic appearance of cystic mediastinal teratoma. 
cular management. Mediastinal teratomas can present unexpectedly as apparent fresh stillbirths. This case highlights the need for full resuscitation in such circumstances, and shows that excellent outcome can be achieved after surgical removal.

ANNELI W ALLMAN

PAUL W BUSS

Department of Child Health, Royal Gwent Hospital, Newport, Gwent, Wales, UK
RICHARD D SPICER

Department of Paediatric Surgery, Bristol Children's

Hospital, Bristol, UK

ANN WAKE

Department of Radiology, Royal Gwent Hospital, Newport

1 Stevens MCG. Neonatal tumours. Arch Dis Child 1988;63:1122-5.

2 Tapper D, Lack EE. Teratomas in infancy and childhood: A 54 year experience at the Children's Hospital Medical Center. Ann Surg 1983;198:398-410. 